TAIWANESE JOURNAL OF MATHEMATICS

Vol. 1, No. 2, pp. 171-180, June 1997

\title{
ON THE CORES OF SCALAR MEASURE GAMES
}

\author{
Man-Chung Ng, Chi-Ping Mo, and Yeong-Nan Yeh
}

\begin{abstract}
A $C V M(k)$ game is a game of the form $f \circ \lambda$, where $\lambda$ is a $k$-dimensional non-atomic measure and $f$ is a continuously differentiable function on $R^{k}$. For a convex $C V M(1)$ game, we characterize the "least upper bound" and "greatest lower bound" of the core elements in terms of the distribution function. We also show that the core of a convex $C V M(1)$ game expands as the underlying measure $\lambda$ changes in a "convex manner". These results provide a partial geometric picture for the core and its variations of a convex $C V M(1)$ game.
\end{abstract}

\section{INTRODUCTION}

A game $V$ with a continuum of players is a bounded real-valued function defined on $\Sigma$, the set of all Borel subsets of $I=[0,1]$, such that $V(\emptyset)=0$. The elements of $\Sigma$ are interpreted as coalitions of players; for each coalition $S, V(S)$ gives the maximum payoff achieved by the efforts of all members in the coalition. With this interpretation, we shall assume that $V$ is non-negative and that $V$ is not identically zero throughout this paper. For $S \in \Sigma$, we denote by $S^{*}$ the indicator function of $S$. Let $\mathbf{B}$ be the Banach space spanned by the set $\Sigma^{*}=\left\{S^{*}: S \in \Sigma\right\}$ with the sup norm. Then the space of all bounded additive functions on $\Sigma$, denoted by $\mathbf{B A}$, is isometrically isomorphic to the norm-dual of $\mathbf{B}$. A payoff of the game $V$ is an element $\mu \in \mathbf{B A}$ with $\mu(I)=V(I)$. The core $C_{V}$ of the game $V$ consists of all payoffs $\mu$ such that no coalition can improve upon, i.e., $\mu(S) \geq V(S)$ for each $S \in \Sigma$. The core is a convex and compact set (in the $\mathbf{B}$ topology). The assumption that $V$ is

Received September 10, 1995

Communicated by G.-J. Chang.

1991 Mathematics Subject Classification: 90D12, 90D13.

Key words and phrases: Convex games, exact games, cores, scalar measure games, vector measure games, core expansion, core geometry 
non-negative implies that any element in the core is also non-negative. In fact, $\mu \in C_{V}$ must be monotonic in the sense that $\mu(S) \leq \mu(T)$ whenever $S \subseteq T$.

Since $\Sigma$ is a $\sigma$-algebra, we can endow a measure $\lambda$ with it ${ }^{1}$. Then a special class of symmetric games (with respect to the measure $\lambda$ ) can be defined as follows:

Definition 1. $V$ is called symmetric (with respect to $\lambda$ ) if $V(S)=V(T)$ for all $S$ and $T$ such that $\lambda(S)=\lambda(T)$.

It is clear that $V$ is symmetric if and only if there is a unique real-valued function $f:[0, \lambda(I)] \longrightarrow R$ such that $V(S)=f \circ \lambda(S)$ for all $S \in \Sigma$, and $f(0)=0$. We shall follow Aumann and Shapley [1974] and call such $V$ a scalar measure game (See Aumann and Shapley [1974], p. 14). More generally, we can define:

Definition 2. Let $\mathbf{0}$ be the origin in $R^{k}$ and $\lambda$ be a $k$-dimensional vector measure on $(I, \Sigma)$ such that its range, denoted by Range $(\lambda)$, is of full dimension in $R^{k}$. The class of all $k$-dimensional vector measure games $V M(k)$ (with respect to $\lambda$ ) consists of all games $V$ of the form: $V(S)=f \circ \lambda(S)$, where $f$ is a non-negative real-valued function defined on $R^{k}$ with $f(\mathbf{0})=0$.

Let $C V M(k)$ be the class of all games $V=f \circ \lambda \in V M(k)$ such that $f$ is continuously differentiable on $R^{k}$, and that $\lambda$ is a non-atomic vector measure ${ }^{2}$ on I. The Shapley value of a game $V=f \circ \lambda \in C V M(k)$ is a payoff $\phi_{V}$ of $V$ given by the following formula (Aumann and Shapley [1974], p. 23)

$$
\phi_{V}(S)=\int_{0}^{1} f_{\lambda(S)}(t \lambda(I)) d t
$$

where $f_{\lambda(S)}$ is the derivative of $f$ in the direction $\lambda(S) \in R^{k}$. In the case $k=1$, the Shapley value is in the core if and only if for each $S \in \Sigma$ such that $\lambda(S)>0, \frac{v(S)}{\lambda(S)}$ is bounded above by $\frac{v(I)}{\lambda(I)}$.

Due to space limitation, the focus of this paper is mostly on the case $k=1$, which carries quite strong geometric intuition. In the second section, we begin with Schmeidler's [1972] result that the core of any convex game consists

\footnotetext{
1 Of particular interest is the Lebsegue measure on $\Sigma$. We also recall that a measure is a non-negative countably additive function in BA.

2 We recall that an atom of a vector measure $\lambda$ is a coalition $S \in \Sigma$ such that $\lambda(S) \neq \mathbf{0}$, and for each proper subset $T$ of $S$, either $\lambda(T)=\mathbf{0}$ or $\lambda(S-T)=\mathbf{0}$. If $\lambda$ has no atom, then it is called a non-atomic measure. It is clear that any measure which is absolutely continuous with respect to the $k$-dimensional Lebsegue measure on $I$ must be non-atomic.
} 
entirely of countably additive elements if the game satisfies some type of continuity condition. It is quite obvious that every convex game in $C V M(1)$ satisfies this condition, so that each core element can be represented by a distribution function. In Section 3, for a convex game in $C V M(1)$, we characterize the "least" upper bound, and the "greatest" lower bound for all the distribution functions in the core. This shows a clear geometric picture for the "shape" of the core. Finally, we prove a theorem on expanding core as we change the underlying measure $\lambda$.

\section{Games in $C V M$}

To every game $V$, we can define an extended real number $|V|$, called the norm of $V$, by

$$
\begin{aligned}
|V|= & \sup \left\{\sum_{i} a_{i} V\left(S_{i}\right):\left(a_{i}, S_{i}\right)\right. \text { is a finite sequence in } \\
& \left.R_{+} \times \Sigma \text { such that } \sum_{i} a_{i} S^{*} \leq I^{*}\right\} .
\end{aligned}
$$

For each game $V$ with finite norm, the exact envelope of $V$ is a game defined by

(3) $\bar{V}(S)=\min \{\mu(S): \mu \in \mathbf{B A}, \mu \geq V$ and $\mu(I)=|V|\}$ for all $S \in \Sigma$.

Definition 3. A game $V$ is called balanced if $|V|=V(I)$. It is called exact if $\bar{V}=V$. It is called convex if $V(S)+V(T) \leq V(S \cup T)+V(S \cup T)$ for all $S, T \in \Sigma$.

It is clear that for any convex game $V=f \circ \lambda \in C V M(1)$, the Shapley value $\phi_{V}(S)=\lambda(S) \frac{V(I)}{\lambda(I)} \geq V(S)$ by convexity of $f$ for each $S \in \Sigma$. Hence, the Shapley value of a convex game in $C V M(1)$ always lies in its core.

Definition 4. A game $V$ is continuous at $S \in \Sigma$ if $V\left(S_{n}\right) \longrightarrow V(S)$ for any monotone sequence $\left\{S_{n}\right\}$ in $\Sigma$ such that $\bigcup_{n} S_{n}=S$. If $V$ is continuous at each $S \in \Sigma$, then $V$ is said to be continuous.

Proposition 1. (Shapley [1971], Schmeidler [1972]) A game V has a nonempty core if and only if it is balanced. Every convex game is exact, and every exact game is balanced.

Proposition 2. (Schmeidler [1972]) Let $V$ be an exact game. Then every element in the core of $V$ is countably additive if and only if $V$ is continuous at $I$. 
Theorem 1. Every exact (hence convex) game in $C V M(k)$ has a nonempty core with countably additive elements only.

Proof. We first prove that every game in $C V M(k)$ is continuous at $I$ for all $k$. Let $V=f \circ \lambda \in C V M(k)$, and $\left\{S_{n}\right\}$ be any monotone sequence in $\Sigma$ such that $\bigcup_{n} S_{n}=I$. Countable additivity of $\lambda$ implies that $\lambda(I)=$ $\lim _{n} \lambda\left(S_{n}\right)$. Continuity (continuous differentiability is not required here) of $f$ implies that $f \circ \lambda(I)=\lim _{n} f \circ \lambda\left(S_{n}\right)$. Hence, the assertion of Theorem 1 follows immediately from Propositions 1 and 2 .

It is easy to show that a game $V=f \circ \lambda \in C V M(1)$ is convex if and only if the derivative $f^{\prime}$ is non-decreasing on $\operatorname{Range}(\lambda){ }^{3}$ The implication of Theorem 1 is that there is a measure on $\Sigma$ such that every element of an exact game in $C V M(1)$ is absolutely continuous with respect to it. We can, then, via the Radon-Nikodym theorem, represent every element in the core by a real-valued function on $I$. In fact, the following theorem shows that for a game $V=f \circ \lambda, \lambda$ is exactly the measure we are seeking.

Theorem 2. Let $V=f \circ \lambda \in C V M(1)$ be an exact game. Then for each $\mu \in C_{V}$, there is a non-negative Borel measurable function $g_{\mu}$ (unique up to the measure $\lambda$ ) such that

$$
\mu(S)=\int_{S} g_{\mu} d \lambda
$$

Proof. Because of Theorem 1 and the Radon-Nikodym theorem, we are left with proving that $\mu$ is absolutely continuous with respect to $\lambda$. We first recall that any element in the core is non-negative since $\mu \geq V \geq 0$. Now, let $S \in \Sigma$ with $\lambda(S)=0$. Then

$$
\mu(I)-\mu(S)=\mu(I-S) \geq V(I-S)=V(I)=\mu(I),
$$

which implies $\mu(S) \leq 0$. It follows that $\mu(S)=0$, and hence $\mu$ is absolutely continuous with respect to $\lambda$.

According to Theorem 2, for an exact game in $C V M(1)$, we may identify each $\mu \in C_{V}$ with its density function $g_{\mu}$. Two measures $\mu_{1}$ and $\mu_{2}$ in the core will always be regarded as equivalent if $\mu_{1}=\mu_{2}$ except on a measurable set $E$, with $\lambda(E)=0$.

Definition 5. A measurable auto-map $\pi$ on I is measure-preserving for $\lambda$ if it is one-one, onto, and $\lambda(S)=\lambda(\pi(S))$ for each $S \in \Sigma$. (Note we have used the notation $\pi(S)=\{\pi(x): x \in S\}$.)

\footnotetext{
${ }^{3}$ Curiously, for $k>1$, there is no corresponding statement in either directions.
} 
It is clear that the set of all measure-preserving maps for $\lambda$ forms a group consisting of idempotent elements only. An easy example of a measurepreserving map for the Lebesgue measure on $I$ is the function $\pi(x)=1-x$. We note that $\pi^{-1}=\pi$.

Theorem 3. Let $\pi$ be any measure-preserving map for $\lambda$. Then for any game $V \in C V M(1), \mu \in C_{V}$ implies $\mu \circ \pi \in C_{V}$. In fact, $g_{\mu \circ \pi}=g_{\mu} \circ \pi$.

Proof. Let $\mu \in C_{V}$ and $\pi$ be a measure-preserving map for $\lambda$. Then for each $S \in \Sigma$, we have

$$
(\mu \circ \pi)(S)=\mu(\pi(S)) \geq V(\pi(S))=V(S) .
$$

Hence, $\mu \circ \pi \in C_{V}$. The second statement follows from:

$$
\int_{S} g_{\mu} \circ \pi d \lambda=\int_{\pi(S)} g_{\mu} d\left(\lambda \circ \pi^{-1}\right)=\int_{\pi(S)} g_{\mu} d \lambda,
$$

where $\pi^{-1}(S)=\{x \in I: \pi(x) \in S\}$.

Theorem 3 has quite concrete geometric interpretation. Furthermore, for any convex game in $C V M(1)$, we can classify the "shape" of the core in terms of distribution functions. This is the main purpose of the next section.

\section{Convex Games in $C V M(1)$}

Definition 6. For any measure $\mu$ on $\Sigma$, one can define the distribution function of $\mu$ on $I$ by $F_{\mu}(x)=\mu([0, x]) \quad$ for $x \in I$.

It can be easily seen that $F_{\mu}$ has the following properties:

(P1) $F_{\mu}$ is a nondecreasing function;

(P2) $F_{\mu}$ is right continuous and has a left-hand limit everywhere;

(P3) $F_{\mu}(0)=0$ and $F_{\mu}(1)=\mu(I)$.

It turns out the converse is also true. Given a distribution function $F$ on $I$ with properties (P1), (P2), $F(0)=0$, and $F(1)$ finite, one can define ${ }^{4}$ a unique measure $\gamma_{F}$ on $\Sigma$ such that $\gamma_{F}((x, y])=F(y)-F(x)$ for any $0 \leq x<y \leq 1$.

Since $\lambda$ is a non-atomic measure on $I, F_{\lambda}$ is a continuous function on $I$. Assuming that $F_{\lambda}$ is strictly increasing, we can define the following auto map on $I$ :

$$
\hat{\pi}(x)=\left\{y \in I: F_{\lambda}(x)+F_{\lambda}(y)=\lambda(I)\right\}, \quad x \in I .
$$

\footnotetext{
4 This is a standard fact in probability theory. See, e.g., Shiryayev [1984].
} 
We now show that $\hat{\pi}$ is a measure-preserving map for $\lambda$. Since $F_{\lambda}(x)=\lambda(I)-$ $F_{\lambda}(\hat{\pi}(x))$, we have $\lambda([0, x])=\lambda([\hat{\pi}(x), 1])$. It can be readily seen that $\lambda(S)=$ $\lambda \circ \hat{\pi}(S)$ for all open intervals $S \in \Sigma$. The collection $\Sigma$ is the smallest $\sigma$ algebra generated by all the open intervals in $I$. By Carathéodory's extension theorem, $\lambda(S)=\lambda \circ \hat{\pi}(S)$ for all $S \in \Sigma$. Since $F_{\lambda}$ is strictly increasing, $\hat{\pi}$ is one-one. Now, $\hat{\pi}(0)=1, \hat{\pi}(1)=0$ and so by the Intermediate Value Theorem, $\hat{\pi}$ is onto as well. It follows that $\hat{\pi}$ is a measure-preserving map for $\lambda$.

For each monotonic game $V \in C V M(1)$, we can define $F_{V}(x)=V([0, x])$ for each $x \in I$. Clearly, $F_{V}$ satisfies properties (P1) to (P3), and there is a unique measure $\gamma_{F_{V}}$ associated with it. Note that $\gamma_{F_{V}}$ is different from $V$ itself unless $V$ is countably additive.

Theorem 4. Suppose that $V=f \circ \lambda$ is a convex game in $C V M(1)$ and that $F_{\lambda}$ is strictly increasing. Then for any $\mu \in C_{V}$, we have

$$
F_{V}(x) \leq F_{\mu}(x) \leq V(I)-F_{V}(\hat{\pi}(x)) \text { for each } x \in I .
$$

Furthermore, the unique measures associated with $F_{V}(x)$ and $V(I)-F_{V}(\hat{\pi}(x))$, respectively, are elements of the core.

Proof. Since a convex game must be monotonic, we know that $F_{V}$ is welldefined. We first prove $\gamma_{F_{V}} \in C_{V}$. It is clear that for each $S, \gamma_{F_{V}}(S)=\int_{S} f^{\prime} d \lambda$. Thus, $\gamma_{F_{V}} \in C_{V}$ if and only if

$$
\int_{S} f^{\prime} d \lambda \geq f(\lambda(S)) \quad \text { for all } S \in \Sigma .
$$

We observe that the collection of all subsets in $\Sigma$ satisfying inequality (6) is closed under finite disjoint union. Now suppose that $S=(x, y]$ for some $0 \leq x \leq y \leq 1$. Then

$$
\begin{aligned}
\int_{x}^{y} f^{\prime} d \lambda & =f \circ F_{\lambda}(y)-f \circ F_{\lambda}(x) \\
& \geq f\left(F_{\lambda}(y)-F_{\lambda}(x)\right) \quad(\text { by convexity and } f(0)=0) \\
& =f \circ \lambda((x, y]),
\end{aligned}
$$

which is exactly inequality (6). Since $\lambda$ is a non-atomic measure, and $f^{\prime}$ is bounded on $[0, \lambda(I)]$, inequality (6) holds for all closed intervals contained in $I$ as well. Hence, inequality (6) is true for any finite disjoint union of closed intervals in $I$. Suppose that for some $S \in \Sigma$, inequality (6) is false. Let

$$
\epsilon=f \circ \lambda(S)-\int_{S} f^{\prime} d \lambda>0 .
$$


Using the facts that $\lambda$ is a non-atomic measure on $\Sigma, f^{\prime}$ is bounded on $I$, and $f$ is continuous, we can find a set $T$ which is a finite disjoint union of closed intervals in $I$ such that

$$
\max \left\{|f \circ \lambda(S)-f \circ \lambda(T)|,\left|\int_{S} f^{\prime} d \lambda-\int_{T} f^{\prime} d \lambda\right|\right\}<\frac{\epsilon}{2} .
$$

This is a contradiction since $f \circ \lambda(T)=\int_{T} f^{\prime} d \lambda$. Hence we have proved that $\gamma_{F_{V}} \in C_{V}$.

By Theorem $3, \gamma_{F_{V}} \circ \hat{\pi} \in C_{V}$. It is easy to check that its distribution function is given by $V(I)-F_{V} \circ \hat{\pi}$.

For the first part of the theorem, let $\mu$ be an element in $C_{V}$. By definition, $F_{\mu}(x) \geq F_{V}(x)$ for each $x \in I$. From Theorem $3, \mu \circ \hat{\pi} \in C_{V}$, and hence

$$
F_{\mu}(x)=V(I)-F_{\mu \circ \hat{\pi}}(\hat{\pi}(x)) \leq V(I)-F_{V}(\hat{\pi}(x)) \text { for each } x \in I .
$$

Definition 7. An element in the core of a game $V$ is called a vertex if it cannot be written as the convex combination of two distinct elements in the core.

According to the above definition, if $V=f \circ \lambda \in C V M(1)$, then both $\gamma_{F_{V}}$ and $\gamma_{F_{V}} \circ \hat{\pi}$ are vertices. The convex hull of these two elements forms part of $C_{V}$. There are also other vertices. In the example below we show a method of constructing some vertices.

Example. Consider the convex game $V=f \circ \lambda$, where $f(x)=x^{2}$ and $\lambda$ is the Lebesgue measure. Then $\hat{\pi}(x)=1-x$ in Theorem 4. All distribution functions in the core are bounded by $y=x^{2}$ and $y=1-f \circ \hat{\pi}(x)=1-(1-x)^{2}$. Now for each $0<a \in I$, let us consider the measure-preserving map $\hat{\pi}_{a}$ for $\lambda$ defined by

$$
\hat{\pi}_{a}(x)= \begin{cases}a-x, & \text { if } x \in[0, a] \\ x, & \text { if } x \in(a, 1] .\end{cases}
$$

Through $\hat{\pi}_{a}$, we obtain the following density function for a distribution function which we call $F_{a}$ :

$$
g_{F_{a}}(x)= \begin{cases}2(a-x), & \text { if } x \in[0, a] \\ 2 x, & \text { if } x \in(a, 1] .\end{cases}
$$

We claim that $F_{a}$ is a vertex of the core. Suppose that there are two elements $F_{1}$ and $F_{2}$ in the core such that $F_{a}=\alpha F_{1}+(1-\alpha) F_{2}$ for some $\alpha \in(0,1)$. Since $F_{1}$ and $F_{2}$ are bounded below by $F_{V}$, we must have $F_{1}(x)=F_{2}(x)=F_{V}(x)$ for all $x \geq a$ (since all distribution functions are right continuous). Since 
$F_{1} \in C_{V}$, the distribution defined by $\bar{F}_{1}=F_{1} \circ \hat{\pi}_{a} \in C_{V}$. Then for each $x \leq a$, we have $\bar{F}_{1}(x)=V(a)-F_{1}\left(\hat{\pi}_{a}(x)\right)$ and hence

$$
F_{1}(x)=V(a)-\bar{F}_{1}\left(\hat{\pi}_{a}(x)\right) \leq V(a)-F_{V}\left(\hat{\pi}_{a}(x)\right)=F_{a}(x) .
$$

Similarly, $F_{2}(x)$ is bounded above by $F_{a}$ on the range $[0, a]$. It follows that $F_{1}=F_{2}=F_{a}$, a contradiction. Hence, $F_{a}$ is a vertex of the core (c.f. Figure $1)$.

Remark. As regards the preceding example, let us consider a finite union of disjoint intervals $E_{i} \subseteq I, i=1,2, \cdots, n$. On each $E_{i}$, one can define a measure-preserving map $\pi_{i}$ by renaming the players of $E_{i}$ in the "opposite" direction. Then define a measure-preserving map $\pi$ on $I$ by letting $\pi=\pi_{i}$ on each $E_{i}$, and $\pi$ be the identity map elsewhere. It is clear that the distribution function with density function $f \circ \pi$ is a vertex.

For each $V=f \circ \lambda$, and each $0<y \in I$, we can define a subgame $V_{y}$ on $[0, y]$ by $V_{y}(S)=V(S)$ for all $S \subseteq[0, y]$ and $S \in \Sigma$. Then, we can easily prove that any element in $C_{V_{y}}$ can be extended to some element in $C_{V_{x}}$ whenever $y \leq x$. The ideas of the proof are clear from Example 1 and the proof of Theorem 4. We now state another theorem in which we change the underlying measure rather than the underlying subspace $[0, y]$.

FIG. 1. 
Theorem 5. (Core Expansion) Let $V_{1}=f \circ \lambda$, and $V_{2}=f \circ \lambda_{2}$ be two convex games in $C V M(1)$. Assume that $F_{\lambda_{1}}$ and $F_{\lambda_{2}}$ are both strictly increasing on $I$, and the function $h=F_{\lambda_{1}}^{-1} \circ F_{\lambda_{2}}$ is convex with $\lambda_{1}(I) \leq \lambda_{2}(I)$. Then, there exists a one-one map $\Phi: C_{V_{1}} \rightarrow C_{V_{2}}$.

Proof. Let $b$ be the unique point in $I$ satisfying $h(b)=1$. Take a distribution $F_{1} \in C_{V_{1}}$. Define the following distribution function:

$$
F_{2}(x)= \begin{cases}F_{1}(h(x)) & \text { if } x \leq b \\ F_{V}(x) & \text { if } x>b\end{cases}
$$

We claim that $F_{2}$ is a distribution in $C_{V_{2}}$. As in the proof of Theorem 4, observing that the collection of all Borel measurable sets satisfying

$$
\gamma_{F_{2}}(S) \geq V_{2}(S) \quad \text { for each } S \in \Sigma
$$

is closed under finite disjoint union, it suffices to prove that for each $0<x \leq$ $y \leq 1$,

$$
F_{2}(y)-F_{2}(x) \geq f\left(F_{\lambda_{2}}(y)-F_{\lambda_{2}}(x)\right) .
$$

Inequality (7) is true from Theorem 4 if both $x, y>b$. Now consider the case when both $x, y \leq b$. In this case,

$$
\begin{aligned}
F_{2}(y)-F_{2}(x) & =F_{1}(h(y))-F_{1}(h(x)) \geq f \circ F_{\lambda_{1}}(h(y)-h(x)) \\
& \geq f \circ F_{\lambda_{2}}(y-x),
\end{aligned}
$$

where the last inequality follows from the convexity of $h$, and the fact that $F_{\lambda_{1}}, F_{\lambda_{2}}$ and $f$ are increasing. To complete the proof, note that when $x \leq$ $b<y,[x, y]$ can be written as the disjoint union of two intervals $[x, b]$, and $(b, y]$. The map $\Phi$, which maps $F_{1} \in C_{V_{1}}$ to $F_{2} \in C_{V_{2}}$ is clearly one-one.

\section{ACKNOWLEDGEMENT}

We would like to thank C. Chen for his capable assistance in this research. Comments and suggestions by two anonymous referees were helpful during the revision process.

\section{REFERENCES}

1. R. J. Aumann and L. S. Shapley, Values of Non-atomic Games, Princeton University Press, 1974. 
2. L. J. Billera and J. Raanan, Cores of non-atomic linear production games, Math. Oper. Res. 6 (1981), 420-423.

3. C. Chen, One Dimensional Symmetric Games With a Continuum of Players, Master thesis, Department of Mathematics, Soochow University, 1995.

4. F. Delbaen, Convex games and extreme points, J. Math. Anal. Appl. 45 (1974). 21-233.

5. E. Einy, R. Holzman, D. Monderer, and B. Shitovitz, Core and stable sets of large games arising in economics, J. Econom. Theory 68 (1996), 200-211.

6. Y. Kannai, Countably additive measures in cores of games, J. Math. Anal. Appl. 27 (1969), 227-240.

7. Y. Kannai, The core and balancedness, in Handbook of Game Theory, 1 (1992), North-Holland.

8. C. P. Mo, M. C. Ng, and Y. N. Yeh, Some results on core geometry in coalitional games, to appear in the Proceedings of the Second International Conference on Applications of Dynamic Models of Economics, National Central University, 1996.

9. D. Schmeidler, On balanced games with infinitely many players, Mimeographed, RM-28, Department of Mathematics, The Hebrew University, Jerusalem, 1967.

10. D. Schmeidler, Cores of exact games, J. Math. Anal. Appl. 40 (1972), 214-225.

11. L. S. Shapley, Cores of convex games, Internat. J. Game Theory 1 (1971), $11-26$.

12. A. Shiryayev, Probability, Springer-Verlag, 1984.

Man-Chung Ng and Chi-Ping Mo

Institute of Economics, Academia Sinica

Nankang, Taipei 11529, Taiwan

Yeong-Nan Yeh

Institute of Mathematics, Academia Sinica

Nankang, Taipei 11529, Taiwan 
Received Received Received Received Received

Received Received Received Received Received

Received Received Received Received Received

Of We Curiously, This 